TEMAS DE ACTUALIDAD

Rev Chil Salud Pública 2019,

Vol 23(1): $68-71$

\section{RELIGIÓN, VUDÚ Y SALUD MENTAL: NUEVOS DESAFÍOS PARA LOS SERVICIOS DE SALUD EN CHILE ${ }^{\circ}$}

\author{
RELIGION, VODOU, AND MENTAL HEALTH: NEW CHALLENGES FOR \\ HEALTH SERVICES IN CHILE.
}

\section{INTRODUCCIÓN}

El arribo de población haitiana a territorio chileno ha traído consigo una serie de desafíos para los servicios de salud del país. Dichos desafíos no se reducen estrictamente a superar las diferencias idiomáticas sino también a integrar, tanto en el diseño de políticas de salud como en la práctica clínica, las denominadas "diferencias culturales". En este contexto, el vudú haitiano ha comenzado a ocupar un lugar central en el trabajo cotidiano de los(as) agentes de salud. Si bien el vudú generalmente evoca un lugar de exotismo asociado a la brujería, éste también puede ser concebido como un sistema de creencias y prácticas que previene y trata problemas de salud, a partir de un cuerpo de conocimientos e intervenciones congruentes que interactúan con otros sistemas de salud occidentales, configurando procesos de salud y enfermedad específicos ${ }^{1,2}$. En tal sentido, las representaciones relativas a las causas y a la experiencia de enfermedad, a las modalidades de intervención y cura, a la clasificación de enfermedades y, por último, a la muerte, tienden a diferir de las representaciones biomédicas de salud y enfermedad imperantes en países occidentales ${ }^{3}$.

Los(as) agentes de salud han enfrentado desafíos específicos ante la emergencia de representaciones vuduistas asociadas a la salud y enfermedad mental. Al comenzar a tratar padecimientos psíquicos cuya configuración se ancla en una visión cosmocéntrica de la realidad ${ }^{4,5}$, los(as) agentes se han visto obligados a interrogar sus propios marcos conceptuales, emanados principalmente desde disciplinas como la psicología, la psiquiatría y la neurología. Adicionalmente, los(as) agentes se han encontrado con pacientes escasamente familiarizados con conocimientos y prácticas provenientes de dichas disciplinas, viendo frustrados frecuentemente sus intentos por establecer un lenguaje común en torno al malestar y el sufrimiento expresado por la población haitiana. De hecho, según un informe publicado el 2003 por la Organización Panamericana de la Salud ${ }^{6}$, Haití contaba en ese entonces con sólo 10 psiquiatras y 9 enfermeros(as) psiquiátricos(as) en su sistema de salud, revelando tanto una escasez de recursos ${ }^{7}$ como una cierta lejanía respecto a las prácticas biomédicas y psicológicas/psiquiátricas preponderantes en otros países de la región. A juicio de algunos investigadores ${ }^{8}$, tal escasez de recursos sería, precisamente, una de las condiciones que permiten al vudú ser un sistema de salud validado por parte de la población haitiana.

El objetivo del presente escrito es hacer una revisión de las principales investigaciones que han dado cuenta de la centralidad del vudú en las representaciones de salud y enfermedad mental presentes en parte del pueblo haitiano, con el fin de re-

a. El presente trabajo se enmarca dentro del proyecto doctoral "Discursos y prácticas de migración y salud mental: una aproximación etnográfica a la subjetividad de jóvenes afrodescendientes en Chile", financiado por BecasChileCONICYT. El autor agradece al Dr. Emmanuel Mompoint por su colaboración en la clarificación de aspectos etnolingüísticos propios de la temática. 
levar su importancia para la salud pública del Chile actual. En un primer momento, describiré al vudú dentro del marco religioso de Haití, así como también su relación con las representaciones de salud y enfermedad mental. Posteriormente, daré cuenta, brevemente, de las formas que tienden a asumir dos cuadros psiquiátricos en población haitiana, dada la importancia que revisten en términos de las políticas de salud en Chile, a saber: psicosis y depresión. Por último, a modo de comentarios finales, destacaré literatura internacional que concibe al vudú como un sistema dinámico que no necesariamente se constituye en un obstáculo para la búsqueda de ayuda psicológica/psiquiátrica o para la efectividad de las intervenciones.

\section{Religión, vudú y salud/enfermedad mental}

La religión es un aspecto central de la vida social en Haití ${ }^{9}$. El catolicismo, el protestantismo, y el vudú predominan en el país, interactuando entre sí a nivel de sus prácticas y símbolos ${ }^{10}$. Dado que el vudú haitiano es un culto que combina tradiciones del África occidental y el catolicismo, la población haitiana católica tiende a ser más receptiva al vudú que la población haitiana protestante ${ }^{11}$. Antes de ser declarada como una religión afro-americana, el vudú era concebido como un conjunto de supersticiones y prácticas catalogadas como "escandalosas". Sin embargo, a partir del trabajo etnográfico de Jean Price-Mars en la década del '20, el mundo académico dejó de lado la connotación "diabólica" del vudú para posicionarlo como una pieza central que da cuenta tanto de los orígenes como de la cotidianidad del pueblo haitiano ${ }^{12}$. Más aún, durante los '60, algunos investigadores comenzaron a catalogar al vudú no sólo como una religión sino que también como un sistema de salud ${ }^{3,11}$.

El vudú, desde una visión cosmocéntrica de la realidad, sitúa al sujeto dentro de un universo compuesto por ancestros, espíritus, y el mundo natural $(\text { "Iwa") })^{3}$. Coherentemente, tanto las concepciones de "persona", "salud", "enfermedad", así como la clasificación de éstas, están compuestas por categorías naturales y supernaturales ${ }^{13,14}$. Según la Organización Mundial de la Salud², parte de la población haitiana concibe a la "persona" a partir de cuatro di- mensiones: "cuerpo sin vida" ("Kò kadav") C, "sombra" ("Lonbraj"), "ángel bueno", ("Gwo bon-anj"), y "angelito bueno" ("Nam/ti bon-anj"). De este modo, la "salud" se encuentra relacionada estrechamente con componentes: (a) no-humanos (plantas, animales, tierra, aire, etc.), (b) humanos (familia nuclear y extensa, redes sociales y colectividades), y (c) espirituales (ancestros, dioses, etc.). Mientras que, por su parte, la "enfermedad" se concibe como la pérdida de armonía entre: (a) los componentes pertenecientes a la persona, o entre (b) la persona y algunos de los componentes no-humanos, ancestrales y/o espirituales.

Por su parte, la clasificación de enfermedades difiere de una visión antropocéntrica ya que contempla enfermedades dentro del: (a) dominio de lo visible o enfermedades físicas ("Maladi Bondyè"), (b) dominio de lo invisible, secreto o mágico ("Maladi fe-moun mal" or "maladi diab"), y (c) dominio espiritual invisible ("Maladi Iwa") 2,3. Comúnmente, las enfermedades alojadas dentro del dominio de lo visible son tratadas por la biomedicina o curanderos tradicionales ("doktèfèy"), mientras que aquellas pertenecientes al dominio de lo invisible y espiritual son tratadas por un mago/brujo practicante del vudú ("bòkò"), un sacerdote ("oungan") o una sacerdotisa ("manbo") vuduista 2,3 . No obstante, la validación social de estos(as) agentes de salud puede variar en función del nivel de estigma que la comunidad asocie al vudú y a las enfermedades mentales ${ }^{15}$. Del mismo modo, la experiencia subjetiva de enfermedad, la búsqueda de ayuda, así como los agentes de salud involucrados varían según el grado de adherencia del paciente al vudú y/o la adscripción a otra religión ${ }^{1,16}$.

El padecimiento psíquico, desde un punto de vista etiológico, puede encontrar su origen tanto en aspectos de la vida personal, familiar y social, así como también en aspectos espirituales. Respecto a estos últimos, las causas de la enfermedad podrían responder, en un primer nivel, a un desequilibrio con el mundo espiritual de los antepasados, mientras que, en segundo nivel, a posesiones espirituales o maldiciones recibidas ${ }^{3}$. Diversas investigaciones han dado cuenta de esto a partir de distintos cuadros psicóticos ${ }^{2}$ y depresivos ${ }^{2}$, así como también a c. En la investigación original de la OMS2, los autores traducen "Kò kadav" al inglés como "body". Sin embargo, "Kò kadav" haría referencia a un
"cuerpo sin vida", mientras que "Kò ak vi" o "moun vivan" darían cuenta de un "cuerpo vivo" en terminología médica 
partir de trastornos asociados a los efectos traumáticos provocados tanto por la violencia en espacios públicos ${ }^{17}$ como por el terremoto que afectó al país el año 201018, ${ }^{19}$. Del mismo modo, algunos estudios se han focalizado en cuadros disociativos o de "trance" 20 , mientras que otros en los denominados síndromes culturales existentes en Haití, tales como el "Sezisman"21, "Endispozisyon"22 o el "Pèdisyon" 23 .

En el marco de este breve escrito abordaré, como mencioné anteriormente, dos cuadros psicopatológicos: psicosis y depresión.

\section{Psicosis}

Una vez que la persona ha presentado repetidos episodios psicóticos y su funcionamiento general se ha visto alterado, el entorno social tiende a concebirlo como una persona que padece de una "enfermedad mental" ("maladi moun fou"), etiquetándolo de "loco" ("fou"). A causa del estigma asociado, el entorno posiblemente no vuelva a confiar en su juicio o capacidades cognitivas, incluso después de largos periodos de remisión ${ }^{15}$. A nivel sintomático, los pensamientos y afectos usualmente son atribuidos a posesiones de espíritus o a maldiciones. Por tanto, es necesario, en términos clínicos, indagar no sólo en la forma de las manifestaciones sino que también en sus contenidos ${ }^{2}$. El bajo número de casos que acceden a atención médica/psiquiátrica en Haití, y que son diagnosticados con la categoría de esquizofrenia, son tratados generalmente con anti psicóticos con el objetivo de eliminar las alucinaciones y los delirios. Por lo tanto, dada la centralidad del vudú en la vida haitiana, en estos casos es de especial relevancia diferenciar entre prácticas vudú que involucran posesión de espíritus -como podría ser el caso de un "trance"-, y los problemas de salud mental que un paciente pueda padecer ${ }^{24}$.

\section{Depresión}

La población haitiana cuenta con dos palabras para hacer referencia a una afección del ánimo. Por un lado, por "depresión" ("dépression") se comprende un estado de desaliento o astenia. Por otro, por "depresión mental" ("dépression mentale") se concibe a los síntomas que componen a la categoría difundida por la psicología y psiquiatría occidental. Por lo general, este cuadro se manifiesta somáticamente con dolores de cabeza, espalda, y dolor corpo- ral inespecífico ${ }^{25}$, así como también con síntomas como insomnio, falta de apetitivo, y sensación de vacío ${ }^{2}$. Desde un punto de vista etiológico, la "dépression mentale" respondería a preocupaciones excesivas de la vida cotidiana o al efecto de una maldición. Del mismo modo, la religión juega un papel relevante en casos que presentan ideaciones y/o intentos de suicidio. Comúnmente, a diferencia de las personas que profesan el catolicismo, quienes poseen creencias vuduistas suelen tener actitudes más favorables hacia el suicidio, ya que tienden a desplazar la culpa y el estigma hacia otras instancias (por ej. una maldición, una "fuerza mística" ("fòs mystik"), entre otras) ${ }^{26}$. En relación a la búsqueda de ayuda de psicológica, la familia extensa brinda apoyo psicológico a quienes se ven afectados por este cuadro, por lo que inusualmente son tratados por profesionales de la salud ${ }^{16,25}$.

\section{Comentarios finales}

El vudú no necesariamente se constituye en un obstáculo para la búsqueda de ayuda psicológica/ psiquiátrica o para la efectividad de las intervenciones $2,3,15$. Más allá de cualquier tipo de culturalismo tendiente a reducir problemáticas sanitarias del pueblo haitiano al vudú, los estudios han recalcado que la escasez de recursos y/o la falta de capacitación entre los agentes de salud podrían ejercer una mayor influencia en las trayectorias de salud de la población haitiana. De esta forma, se vuelven prioritarias estrategias como la capacitación de agentes en salud mental así como también la formación de mediadores interculturales. Ambas estrategias han sido ampliamente validadas en diversos contextos $^{27}$. Del mismo modo, se torna necesario promover estudios locales que anuden problemáticas como religión, espiritualidad y salud (mental), tal como diversas investigaciones internacionales han desarrollado a partir de los fenómenos migratorios contemporáneos ${ }^{28,29}$.

\section{REFERENCIAS BIBLIOGRÁFICA}

1. Vonarx N. Vodou, illness and models in Haiti: From local meanings to broader relations of domination. Anthropology in Action. 2007; 14(3): 18-29.

2. WHO/PAHO. Culture and mental health in Haiti: A Literature review. Geneva: WHO; 2010 
3. Vonarx N. Le Vodou haïtien. Entre medicine, magie et religion. PUR: Rennes; 2012

4. Sterlin C. Pour une approche interculturelle du concept de santé. Ruptures, 2006; 11(1): 112-121.

5. Farmer P. The birth of the Klinik: A cultural history of Haitian professional psychiatry. En: Gaines A., editor. Ethnopsychiatry: The cultural construction of professional and folk psychiatries. Albany: SUNY; 1992. p. 251-272.

6. PAHO. Haiti: Profile of the health services system. Washington DC: PAHO; 2003.

7. Nicolas G, Jean-Jacques R, Wheatley A. Mental health counseling in Haiti: Historical overview, current status, and plans for the future. Journal of Black Psychology, 2012, 38(4): 509-519.

8. Wagenaar B, Kohrt B, Hagaman A., et al. Determinants of care seeking for mental health problems in rural Haiti: Culture, cost, or competency. Psychiatric Services, 2013 64(4): 366-372.

9. Dubois L. Haiti: the aftershocks of history. New York: Metropolitan Books; 2012.

10. Hurbon L. Pentecostalism and transnationalism in the Caribbean. En: Corten A, et al. editores. Between Babel and Pentecost: Transnational Pentecostalism in Africa and Latin America. London: Hust \& Co; 2001. p. 124-41.

11. Métraux A. Le Vaudou Haïtien. Paris: Gallimard; 1958.

12. Byron JP. La pensée de Jean Price-Mars. Entre construction politique de la nation et affirmation de l'identité culturelle haïtienne. En: Byron JP, editor. Production du savoir et construction sociale. L'ethnologie en Haïti. Québec/Por-au-Prince: Les Presses de I'Université Laval \& Les Éditions de l'Université d'État d'Haïti; 2014. p. 47-80

13. Coreil J. Parallel structures in professional and folk health care: a model applied to rural Haiti. Cult Med Psychiatr, 1983 7(2), 131-151.

14. Kiev A. Folk psychiatry in Haiti. J of Nerv Ment Dis, 1961; 1322: 260-265.

15. Khoury N, Kaiser B, Keys H, et al. Explanatory models and mental health treatment: Is Vodou an obstacle to psychiatric treatment in rural Haiti? Cult Med Psychiatry, 2012, 36:514-534.

16. Desrosiers A, Fleurose S. Treating Haitian patients: key cultural aspects. American Journal of Psychotherapy, 2002, 56(4), 508-521.

17. Bolton P, Surkan P, Gray A., et al. The mental health and psychosocial effects of organized violence: A qualitative study in northern Haiti. Transcultural Psychiatry, 2012 49(3-4): 590-612.

18. Raviola G, Severe J, Therosme T, et al. The 2010 Haiti earthquake response. Psychiatr Clin North Am; 2013 36(3):431-50.

19. Cénat JM, Derivois D. Assessment of prevalence and determinants of posttraumatic stress disorder and depression symptoms in adult survivors of earthquake in Haiti after 30 months. J Affect Disord, 2014, 159:1117.

20. Cavanna AE, Cavanna S, Cavanna A. Epileptic seizures and spirit possession in Haitian culture: report of four cases and review of the literature. Epilepsy Behav, 2010, 19(1):89-91.

21. Nicolas G, DeSilva AM, Grey KS, et al. Using a multicultural lens to understand illness among Haitians living in America. Professional Psychology: Research and Practice, 2006, 37(6), 702-707.

22. Philippe J, Romain JB. Indisposition in Haiti. Social Science and Medicine, 1979, 13B(2), 129-133.

23. Coreil, J, Barnes-Josiah DL, Augustin A, et al. Arrested pregnancy syndrome in Haiti: findings from a national survey. Medical Anthropology Quarterly, 1996, 10(3), 424-436.

24. Azaunce M. Is it schizophrenia or spirit possession? Journal of Social Distress and the Homeless, 1995, 4, 225-263.

25. Hillel J, Desrosiers P, Turnier L. La dépression chez I'Haïtien. Médecin du Québec, 1994, 29(2): p. 121 123.

26. Hagaman A, Wagenaar B, McLean K, et al. Suicide in rural Haiti: clinical and community perceptions of prevalence, etiology, and prevention. Soc Sci Med. 2013, 83:61-9.

27. Collazos F, Qureshi A. La diversidad cultural y la salud mental en el dispositivo hospitalario. En: Comelles J, et al. editores. Migraciones y salud. Tarragona: Publicaciones URV; 2009.

28. Baetz M, Toews J. Clinical implications of research on religion, spirituality, and mental health. Can J. Psychiatry, 2009, 54(5): 292-301.

29. Boehnlein J. Religion and spirituality in psychiatric care: Looking back, looking ahead. Transcultural Psychiatry, 2006, Vol 43(4): 634-651. 